\title{
Observation of self-organized filaments in a dielectric barrier discharge of Ar gas
}

\section{$\operatorname{AUTHOR}(\mathrm{S})$ :}

Shirafuji, Tatsuru; Kitagawa, Takayuki; Wakai, Tatsuro; Tachibana, Kunihide

\section{CITATION:}

Shirafuji, Tatsuru ... [et al]. Observation of self-organized filaments in a dielectric barrier discharge of Ar gas. APPLIED PHYSICS LETTERS 2003, 83(12): 2309-2311

\section{ISSUE DATE:}

2003-09-22

URL:

http://hdl.handle.net/2433/50511

\section{RIGHT:}

Copyright 2003 American Institute of Physics. This article may be downloaded for personal use only. Any other use requires prior permission of the author and the American Institute of Physics. 


\title{
Observation of self-organized filaments in a dielectric barrier discharge of Ar gas
}

\author{
Tatsuru Shirafuji ${ }^{\mathrm{a})}$ \\ International Innovation Center, Kyoto University, Yoshida-Honmachi, Sakyo-ku, Kyoto 606-8501 Japan \\ Takayuki Kitagawa, Tatsuro Wakai, and Kunihide Tachibana \\ Department of Electronic Science and Engineering, Kyoto University, Yoshida-Honmachi, Sakyo-ku, \\ Kyoto 606-8501 Japan
}

(Received 24 February 2003; accepted 23 July 2003)

\begin{abstract}
Symmetric self-organized discharge filaments have been observed in the $\mathrm{rf}(500 \mathrm{kHz})$ dielectric barrier discharge of $\mathrm{Ar}$ gas between two parallel quartz plates with a $\mathrm{MgO}$ film. The arrangement of the filaments is confined around the center on the quartz plate plane. With increasing voltage, the number of filaments increases, and the area of filament arrangement also increases. The arrangement of the filaments does not move if the quartz plate with a $\mathrm{MgO}$ film is employed, while the whole arrangement rotates without a $\mathrm{MgO}$ film. According to the results of current-voltage measurements, Lorentz attractive force is much smaller than Coulomb repulsive force. This suggests that a confinement potential exists as in the case of two-dimensional Coulomb systems in a parabolic potential. However, some of the filament arrangements do not match to those for charged particles in the Coulomb systems, which suggests that the confinement potential does not have pure parabolic profile. (C) 2003 American Institute of Physics. [DOI: 10.1063/1.1613796]
\end{abstract}

Dielectric barrier discharges (DBDs) have been known to be sustained mostly by filamentary streamers when they are operated under high $p d(>500$ Torr $\mathrm{cm})$ product of the gas pressure $p$ and the distance $d$ between electrodes. Several researchers have found that the filamentary discharges can be seen even for lower $p d$ conditions, in which selforganized arrangements of the filaments have been observed. ${ }^{1-6}$ A mechanism for spontaneous filament formation have already been reported by Brauer et al., in which appearance of equally separated filaments can be explained in terms of electron and ion transport coefficients. ${ }^{4}$ On the other hand, motion of the filaments is not fully understood yet, although motion of a pair of filaments has been analyzed by Brauer et al. ${ }^{5}$

In this work, we report on quite symmetric and fixed self-organized arrangements of a few filaments confined in a center of the DBD area of Ar gas. Although surface effects are reported to be negligible according to the Brauer's model, ${ }^{4}$ we report a possibility to fix position of the filaments using a $\mathrm{MgO}$ film evaporated on a dielectric electrode for DBDs. Observed filament arrangements are quite similar to so-called two dimensional (2D) Coulomb systems in which charged particles form self-organized arrangements in an external confinement potential. ${ }^{7-11}$ We have compared our results to those for the 2D Coulomb systems.

Figure 1 shows schematic representation of our experimental setup, which consists of two quartz plates with diameter $80 \mathrm{~mm}$ and thickness $1.5 \mathrm{~mm}$. An indium tin oxide (ITO) film has been evaporated on each quartz plate as a transparent electrode, and its diameter and thickness is 60 $\mathrm{mm}$ and $150 \mathrm{~nm}$, respectively. Distance between the two dielectric plates was $4 \mathrm{~mm}$. The bottom electrode was

${ }^{a)}$ Electronic mail: shira@iic.kyoto-u.ac.jp grounded, and the top electrode was connected to a rf (500 $\mathrm{kHz}$ ) power source (RF Power Products, LF-5) through an automatic matching controller (ASTECH Corp., TL-10R with RC-11). A MgO film (100 nm thick) was evaporated at $250{ }^{\circ} \mathrm{C}$ on the discharge side of the top electrode. Applied voltage and current were monitored by a digital oscilloscope (Iwatsu-LeCroy LT344 500MHz) through a high-voltage probe (Tektronix P6015A) and ac current probe (Tektronix P6021). Optical emission was also monitored by a photomultiplier. Our experimental procedure was as follows; the reactor was once pumped to $10^{-3}$ Torr, and ignition of discharge was performed at Ar pressure of 60-100 Torr and voltage (amplitude) around $1 \mathrm{kV}$. After the ignition, Ar pressure was increased to 210 Torr. Observation of discharge filaments was performed through the top electrode and quartz window.

Figures 2(a)-2(f) show the arrangements of one to six filaments, which possess quite symmetric self-organized arrangements. The number of filaments increased with increasing voltage from 1 to $1.07 \mathrm{kV}$. Variation of the filament ar-

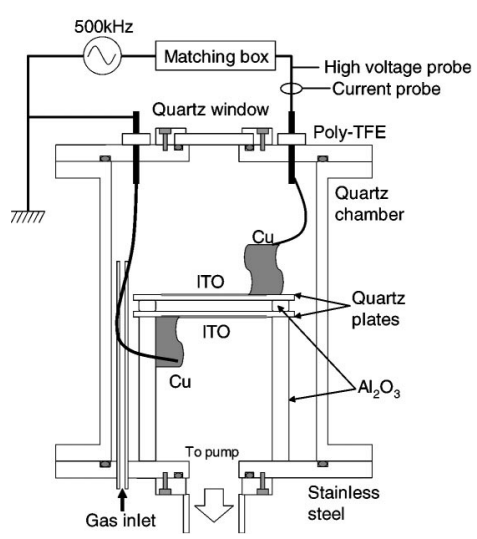

FIG. 1. Experimental setup for observation of filaments in a DBD of Ar gas. 

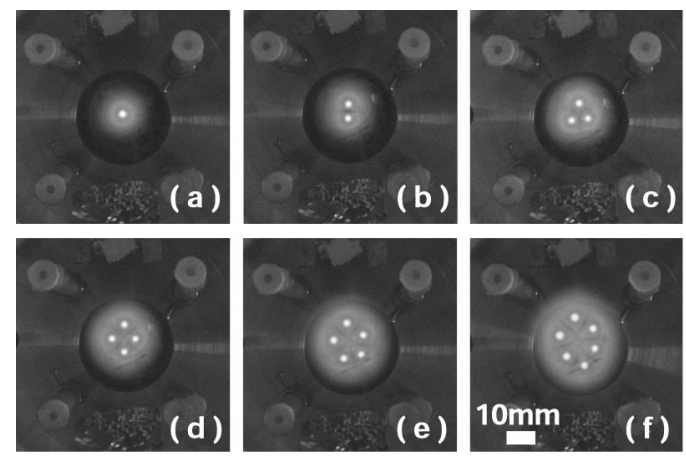

FIG. 2. Arrangements of filaments observed with increasing applied voltage (amplitude). (a) 1, (b) 1.01, (c) 1.02, (d) 1.03, (e) 1.05 , and (f) $1.07 \mathrm{kV}$.

rangements was triggered by quite small change in the voltage. Current and voltage wave forms showed no noticeable variation with increasing the voltage. The area of discharge was expanded with increasing the number of filaments. Due to this expansion, averaged distance between two adjacent filaments was almost fixed and was approximately $7 \mathrm{~mm}$.

Typical current and voltage wave forms in our experiments are shown in Fig. 3(a), which corresponds to the arrangements of 22 filaments. It is known that a conventional DBD current wave form has sharp pulse components corresponding to random filamentary discharges. In this figure, however, the current wave form is close to a simple sine wave form. Phase difference between the current and voltage is almost $90^{\circ}$. This means that most of the current is for charging the whole electrodes. A pure sine wave form component was subtracted from the experimental current wave form in order to extract the discharge current shown in Fig. 3(b). The optical emission peaks in the same figure are in phase with the discharge current peaks. In addition, each current peak is not ensemble of sharp peaks as in conventional DBDs, but one broad peak. This means that each filament is not generated randomly but in phase.

Although Brauer et al. have already presented two-
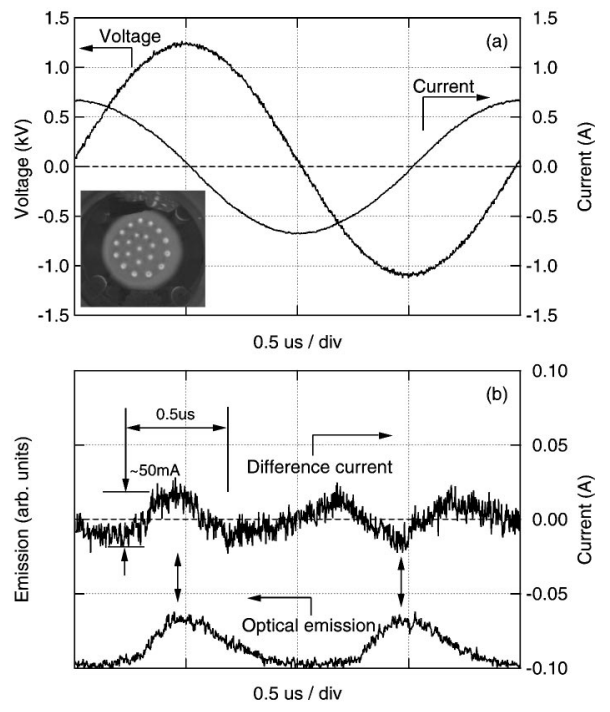

FIG. 3. (a) Current-voltage characteristics of our DBD during discharge with self-organized 22 filaments. (b) Difference current wave form, which has been obtained by subtracting fitted sine wave from measured current wave form, and optical emission wave form. because voltage on the ITO film is not applied uniformly but
Downloaded 04 Mar 2008 to 130.54.110.22. Redistribution subject to AIP license or copyright; see http://apl.aip.org/apl/copyright.jsp dimensional numerical simulation of spontaneous filament formation, ${ }^{4}$ a three-dimensional simulation is required for explaining the present geometric arrangement of filaments. Instead, we have compared our results to those for 2D Coulomb systems. ${ }^{12-16}$

On the surface of dielectric electrodes, there is negative or positive charge for one discharge filament. Its sign depends on the phase of discharge current. As the charge is localized around the position of the filament due to slow mobility on the insulator surface, a filament is always accompanied with charge on the surface, which brings about a repulsive force between the filaments.

For establishing balanced condition for the filaments, there must be an attractive force between the filaments and/or potential which can confine the filaments. Lorentz force between each filament current works as an attractive force. However, its value cannot be balanced as described next. As shown in Fig. 3(b), total filament current is approximately $50 \mathrm{~mA}$ for 22 filaments. Therefore, current for one filament is $I=2.3 \mathrm{~mA}$. Duration of the current is approximately $t=0.5 \mu \mathrm{s}$ according to Fig. 3(b). Maximum charge on the surface is estimated to be $Q=I t=1.15 \mathrm{nC}$, where the current is assumed to be constant for simplifying the present model. The Coulomb force between two charged regions can be estimated from the following equation:

$$
\boldsymbol{F}_{\mathrm{Q}}(i, j)=\frac{1}{4 \pi \epsilon_{0}} \frac{Q^{2}}{\left|\boldsymbol{r}_{i}-\boldsymbol{r}_{j}\right|^{3}}\left(\boldsymbol{r}_{i}-\boldsymbol{r}_{j}\right),
$$

where $\boldsymbol{r}_{i}$ and $\boldsymbol{r}_{j}$ are the positions of a pair of filaments, and $\left|\boldsymbol{r}_{i}-\boldsymbol{r}_{j}\right|=7 \mathrm{~mm}$ as mentioned earlier. $\boldsymbol{\epsilon}_{0}$ is dielectric constant of vacuum. Absolute value of $\boldsymbol{F}_{\mathrm{Q}}(i, j)$ becomes $2.42 \times 10^{-4}$ $\mathrm{N}$. On the other hand, the Lorentz attractive force between a pair of filament current can be estimated from the following equation:

$$
\boldsymbol{F}_{\mathrm{I}}(i, j)=-\frac{\mu_{0}}{2 \pi} \frac{I^{2}}{\left|\boldsymbol{r}_{i}-\boldsymbol{r}_{j}\right|^{2}}\left(\boldsymbol{r}_{i}-\boldsymbol{r}_{j}\right),
$$

where $\mu_{0}$ is permeability of vacuum. Absolute value of $\boldsymbol{F}_{\mathrm{I}}(i, j)$ becomes $1.51 \times 10^{-10} \mathrm{~N}$, which is much weaker than the Coulomb force. This means that a certain confinement potential must exists as employed in a model of 2D Coulomb systems. $^{7-11}$

A model of a 2D Coulomb system consists of charged particles and a confinement potential which is mostly parabolic one. According to the numerical simulation by Bedanov and Peeters, arrangement of $N$ particles has a shell structure $\left(N_{1}, N_{2}, \ldots\right)$ where $N_{i}$ indicates number of particles on each shell and $N=N_{1}+N_{2}+\ldots{ }^{13}$ In their report, pentagonal (1,5)-arrangement should be the most stable ground-state arrangement. In the case of our experiments, however, hexagonal (6)-arrangement always appears as shown in Fig. 2(f). This (6)-arrangement is a metastable state according to Bolton and Rössler, ${ }^{12}$ and observed by Saint Jean et al. ${ }^{14}$ According to the detailed analysis of the metastable arrangement of the 2D Coulomb system by Kong et al., a metastable arrangement for pure parabolic potential can appear as a ground-state arrangement if the potential profile deviate from pure parabolic profile. ${ }^{15}$ This might be a reason why $(1,5)$-arrangement does not appear in our system, 
partially as in Fig. 1, and ITO does not have high conductivity like metals which leads complex electric field between two the quartz electrodes.

If we can apply this model, all the filaments must appear in phase for fixing their relative position. Otherwise, the Coulomb repulsive force between a set of two filaments becomes different because amount of the surface charge is different for each filament, and the behavior of the filaments become as in conventional random-streamer discharges. As mentioned earlier, in our experiments, all the filaments appear in phase according to the observation of temporal behavior of optical emission. Therefore, the model used in the Coulomb system can be applied to explaining our experimental results.

In the case of discharge using the electrode with a $\mathrm{MgO}$ film on the side of discharge area, the position of these filaments was fixed except for the sequence when a streamer was added or removed by changing voltage. This phenomenon was reproducible. For comparison, the dielectric electrode without the $\mathrm{MgO}$ film was also examined. In this case, the arrangement of filaments was almost the same to the results with the $\mathrm{MgO}$ film. However, the arrangement of the filaments was rotating in the clockwise direction with the speed of approximately $1 \mathrm{rps}$. $\mathrm{MgO}$ films in DBDs are known to have an effect of reducing ignition and sustain voltage for the DBDs because of their high secondary electron emission rate. ${ }^{17,18}$ This might be one of the causes to stop the rotation of streamer arrangements because residual ions in the previous discharge can promote the next discharge at the same position. Although it is reported that surface effects can be neglected, ${ }^{4}$ the result obtained here sug- gests that possibility to fix the position of self-organized filaments by using the surface effects.

This research was partially supported by the Kyoto Nanotechnology Cluster.

${ }^{1}$ E. Ammelt, D. Schweng, and H.-G. Purwins, Phys. Lett. A 179, 348 (1993).

${ }^{2}$ I. Muller, E. Ammelt, and H.-G. Purwins, Phys. Rev. Lett. 82, 3428 (1999).

${ }^{3}$ I. Muller, C. Punset, E. Ammelt, H.-G. Purwins, and J. P. Boeuf, IEEE Trans. Plasma Sci. 27, 20 (1999).

${ }^{4}$ I. Brauer, C. Punset, H.-G. Purwins, and J. P. Boeuf, J. Appl. Phys. 85, 7569 (1999).

${ }^{5}$ I. Brauer, M. Bode, E. Ammelt, and H.-G. Purwins, Phys. Rev. Lett. 84, 4104 (2000).

${ }^{6} \mathrm{~K}$. H. Schoenbach and K. Becker, presented at the 3rd International Workshop on Basic Aspects of Non-equilibrium Plasmas Interacting with Surfaces-The New World of Microplasmas, Awaji Yumebutai International Conference Center, Kyoto, Japan, 2003 (unpublished).

${ }^{7}$ L. Candido, J. P. Rino, N. Studart, and F. M. Peeters, J. Phys.: Condens. Matter 10, 11627 (1998).

${ }^{8}$ J. M. Liu, W. T. Juan, J. W. Hsu, Z. H. Huang, and I. Lin, Plasma Phys. Controlled Fusion 41, A47 (1999).

${ }^{9}$ A. Piel and A. Melzer, Plasma Phys. Controlled Fusion 44, R1 (2002).

${ }^{10}$ Y. J. Lai and I. Lin, Phys. Rev. E 60, 4743 (1999).

${ }^{11}$ H. Totsuji, T. Kishimoto, and C. Totsuji, Phys. Rev. Lett. 78, 3113 (1997).

${ }^{12}$ F. Bolton and U. Rössler, Superlattices Microstruct. 13, 139 (1993).

${ }^{13}$ V. M. Bedanov and F. M. Peeters, Phys. Rev. B 49, 2667 (1994).

${ }^{14}$ M. Saint Jean, C. Even, and C. Guthmann, Europhys. Lett. 55, 45 (2001).

${ }^{15}$ M. Kong, B. Partoens, and F. M. Peeters, Phys. Rev. E 65, 046602 (2002).

${ }^{16}$ M. Marlo, M. Alatalo, A. Harju, and R. M. Nieminen, Phys. Rev. B 66, 155322 (2002).

${ }^{17}$ J. P. Boeuf, J. Phys. D 36, R53 (2003).

${ }^{18}$ Y. Motoyama, H. Matsuzaki, and H. Murakami, IEEE Trans. Electron Devices 48, 1568 (2001). 